\title{
Stability change of intrinsic localized mode in finite nonlinear coupled oscillators
}

$\operatorname{AUTHOR}(\mathrm{S})$ :

Kimura, Masayuki; Hikihara, Takashi

\section{CITATION:}

Kimura, Masayuki ... [et al]. Stability change of intrinsic localized mode in finite nonlinear coupled oscillators. Physics Letters A 2008, 372(25): 4592-4595

\section{ISSUE DATE:}

2008-06

URL:

http://hdl.handle.net/2433/85167

\section{RIGHT:}

Copyright (C) 2008 Elsevier B.V.; This is not the published version. Please cite only the published version.; この論文は出版社版でありません。引 用の際には出版社版をご確認ご利用ください。 


\title{
Stability Change of Intrinsic Localized Mode in Finite Nonlinear Coupled Oscillators
}

\author{
Masayuki Kimura $^{\text {a }}$, Takashi Hikihara ${ }^{a}$ \\ ${ }^{a}$ Department of Electrical Engineering, Kyoto University, Nishikyo, Kyoto \\ 615-8510 Japan
}

\begin{abstract}
Intrinsic localized mode (ILM) is spatially localized and temporally periodic oscillation in nonlinear coupled oscillators. We numerically investigate the dynamical stability of ILMs in a microcantilever array, in which ILMs were experimentally observed by M. Sato et al. It is found that the stability change of ILMs is due to the ratio in nonlinear potentials. This phenomenon also occurs in the array without harmonic potentials. Consequently, the stability of ILMs substantially depends on the ratio in nonlinear on-site and inter-site potentials.
\end{abstract}

Key words: Intrinsic localized mode, Discrete breather, Coupled cantilever array, Stability

PACS: 05.45.-a

\section{Introduction}

In 1988, Sievers and Takeno theoretically found a localized mode in an anharmonic lattice [1]. The mode is spatially localized and temporally periodic solution, which is called intrinsic localized mode (ILM) or discrete breather 
(DB). Tremendous number of analytical and numerical studies have been reported. Most of the studies are the rigorous proof of existence, the investigation on dynamical stability and movability, and the development of computational method to obtain ILMs $[2,3]$.

In this decade, experimental investigations have been also reported. ILM is generated and observed in various systems, for instance, Josephson-junction array [4-6], optic wave guides [7,8], and micro-mechanical oscillators [9]. They provide us the generality of localizing phenomena and the possibility of applications as a localized exciter. In fact, the studies toward future applications are increasing both in fundamental science and in practical engineering [10].

Intrinsic localized mode in a micro-mechanical cantilever array has been observed by Sato et al. $[9,11]$. They fabricated a di-elements cantilever array via micro-electro mechanical system (MEMS) technology. The array consisted of short and long cantilevers which were alternatively arranged in one dimension. A piezo vibrator was attached to the substrate of the array. The frequency of the vibrator was chirped to excite ILMs so that ILMs were excited through the modulational instability. As a result, traveling ILMs and pinned ILMs appeared $[11,12,9]$.

Moreover, Sato et al. achieved a shift of the position of ILM using a local impurity $[13,12]$. Thus ILM was possibly shifted from a site to another without any decay. It suggests the possibility of applications of pinned and traveling ILMs into MEMS devices. Then the analysis of dynamical properties of ILM is essential to develop applications.

This Letter focuses on the stability of ILM in the cantilever arrays numerically. In particular, the dependency of the stability of ILMs is investigated. 


\section{Micro-Cantilever Array}

A finite cantilever array is mainly considered in this Letter. The schematic configuration is shown in Fig.1. For simplicity, we assume the mono-element cantilever array. The array has eight homogeneous cantilevers placed with equal interval. Adjacent cantilevers are coupled together by the overhang part between them. In our setting condition, both ends of the array are fixed by the support.

The vibration of the tip of cantilevers is approximately described by the coupled non-dimensional ordinary differential equations as follows:

$$
\begin{aligned}
\ddot{u}_{i}= & -u_{i}-u_{i}^{3}-\alpha\left(2 u_{i}-u_{i-1}-u_{i+1}\right) \\
& -\beta\left(u_{i}-u_{i-1}\right)^{3}-\beta\left(u_{i}-u_{i+1}\right)^{3}, \\
& i \in\{1,2, \ldots, 8\},
\end{aligned}
$$

where $u_{i}$ denotes the displacement of tip of the $i$ th cantilever from equilibrium position. $\alpha$ depicts the ratio in quadratic potentials. The ratio of quartic potential is given as $\beta$. Here we introduce the boundary conditions as fixed-ends; $u_{0}=u_{9}=0, \dot{u}_{0}=\dot{u}_{9}=0$. The experiments by Sato et al. were performed in high vacuum condition [9]. Then we assume that the energy dissipation is negligible. In this Letter, the external exciter is also neglected because the array has no energy dissipation. Hence the total energy of the discretized model

$$
H=\sum_{i}\left\{\frac{1}{2} \dot{u}_{i}^{2}+\frac{1}{2} u_{i}^{2}+\frac{1}{4} u_{i}^{4}+\frac{\alpha}{2}\left(u_{i}-u_{i-1}\right)^{2}+\frac{\beta}{4}\left(u_{i}-u_{i-1}\right)^{4}\right\}
$$

will be kept during the temporal development. The first term is the kinetic energy of a site in the discretized model. The other terms represent the potential energy. On-site potentials are represented by the second and the third 
terms. The forth and the last term are inter-site potentials. They are represented with coefficients $\alpha$ and $\beta$. In this Letter, the linear coupling coefficient $\alpha$ is fixed at 0.1 or set to zero. The quartic interaction coefficient is varied in this Letter.

In this Letter, an ILM is treated as a fixed point on a hyperplane $\Sigma_{4}$. The hyperplane is given by

$$
\Sigma_{i}=\left\{\left(u_{1}, \ldots, u_{8}, \dot{u}_{1}, \ldots, \dot{u}_{8}\right) \in \mathbf{R}^{16} \mid u_{i}>0, \dot{u}_{i}=0\right\}
$$

\section{Coexistence and Stability}

In this section, we discuss the coexistence and the stability of ILMs. The Newton-Raphson method started with an appropriate initial condition is applied to obtain each ILM. Eq.(1) is integrated by 6th-order symplectic integrator. The total energy $H$ is set at 2.5 so that ILM is sufficiently localized. The accuracy of the numerical simulation is confirmed through the preservation of the total energy $H$ with keeping numerical errors under $\epsilon H$. In this Letter, $\epsilon=10^{-12}$.

\subsection{Coexisting ILMs}

The coupled cantilever array has many coexisting ILMs. The symmetry of ILM generally classifies ILMs into two kinds, "Sievers-Takeno mode (ST-mode)" and "Page mode (P-mode)" [2]. The amplitude distribution of the ST-mode illustrated in Fig.2(a) is symmetric in space and centered on a site. This mode is found analytically by Sievers and Takeno [1]. On the other hand, the P- 
mode, which is analytically derived in 1990 [14], is anti-symmetric and centered between sites as shown in Fig.2(b). Here we distinguish coexisting ILMs by index number of cantilever at which the center of each ILM stands. For example, ST4 implies the ST-mode centered on the 4th cantilever and P2-3 implies the P-mode centered between the 2nd and the 3rd cantilevers.

If an ILM is sufficiently localized, it will be able to stand at any site even near the edges. However, the number of coexisting ILMs depends on $\beta$. For $\beta=0.1$, eight ST-modes and seven P-modes are obtained. ST1, ST2, P1-2, and P2-3 are disappeared, when $\beta$ increases up to 0.5 . P2-3 is obtained again at $\beta=0.6$.

\subsection{Stability Change}

The stability of each coexisting ILMs is also changed between $\beta=0.5$ and 0.6. All ST-modes are stable and all P-modes are unstable when $\beta$ is set at 0.5. In contrast, the stability of all coexisting ILMs is flipped at $\beta=0.6$. Fig.3(a) shows locus of coexisting ILMs and their stability with respect to $\beta$. The locus of ILM is obtained by $X_{\Sigma_{4}}=\sum_{i=1}^{8} i\left|u_{i}\right| / \sum_{i=1}^{8}\left|u_{i}\right|$, where $u_{i}$ denotes the displacement of $i$ th cantilever on the hyper plane $\Sigma_{4}$. The linear potential ratio $\alpha$ is fixed at 0.1 . As in Fig.3(a), the stability of P4-5, ST4, and P3-4 is flipped between $\beta=0.5452$ and $\beta=0.5456$.

The locus of stable ST3 is shifted from 3.0 toward 3.5 with $\beta$. Ultimately stable ST3 and unstable P3-4 are disappeared. Unstable ST3 appears with stable P23. The locus of stable P2-3 asymptotically reaches 2.5 as $\beta$ increases. Thus ST3 has a parameter gap between stable and unstable modes. 
Because of the reflectional symmetry about the center of array, only P4-5 changes its stability without any discontinuity. Unstable and stable P4-5 continuously join at $\mathrm{B}_{\mathrm{P}}$. Two stable ST-modes join with $\mathrm{P} 4-5$ and disappeared at the same condition. $\mathrm{P} 4-5$ changes only its stability at $\mathrm{B}_{\mathrm{P}}$.

The absolute value and argument of the Floquet multipliers for P4-5 are shown in Figs. 3(b) and (c). Two Floquet multipliers lie on real axis for $\beta<\beta_{\mathrm{P}}$. One of them exists outside unit circle. The other stays inside unit circle. According to the increase of $\beta$, two Floquet multipliers slide toward +1 along real axis. At $\beta=\beta_{\mathrm{P}}$, these multipliers are conjoined at +1 . After the bifurcation, their multipliers slide on unit circle. Therefore the bifurcation of ILMs on $\beta$ is classified to "saddle-node bifurcation" [15].

\subsection{Analysis on Ringed Array}

Figure 3(a) shows that coexisting ILMs have a parameter gap between stable and unstable modes in $\beta$. The parameter gap tends to be wider with the locus of ILM approaching to the edge. The reason is that the amplitude near the edges increases when ILM approaches to the edges. Then the influence of the edges becomes remarkable. In a ringed array, all coexisting ST-modes and P-modes show their continuous connection to the corresponding ILMs. The numerical confirmation is obtained in Fig.4(a). The parameter gaps are completely vanished. Hence it is concluded that the parameter gap between stable ILM and unstable ILM is caused by the fixed-ends, namely, the effect of the impurities.

The ringed array has translational symmetry. Then all of the P-modes simul- 
taneously change its stability at $\beta_{\mathrm{P}}$ as shown in Fig.4(a). The stability of the ST-modes also changes at $\beta=\beta_{\mathrm{ST}}$. As can be seen from Fig.4(a), the critical values $\beta_{\mathrm{P}}$ and $\beta_{\mathrm{ST}}$ are different. This case corresponds to $\beta_{\mathrm{P}}>\beta_{\mathrm{ST}}$. Thus there exists a region in which all of the coexisting P- and ST-modes are unstable. The region is darkly hatched in Fig.4(a). In the region, asymmetric ILMs, which are represented by dash-dotted curve, also coexist with P- and ST-modes. The amplitude distribution is shown in Figs.4(b) and (c). These asymmetric ILMs are stable and stand between P- and ST-modes. Then they cannot be classified into P- or ST-mode. It should be noted that the region is not affected by lattice number. It is numerically confirmed that the region exist in case of $N=256$.

Figure 4(a) is obtained by the high energy limit, which is achieved by eliminating harmonic terms from Eq.(1). If the array has harmonic potentials, the region is shifted with the total energy $H$. Critical values in Fig.3 decrease according to the decrease of the total energy $H$. Ultimately, coexisting ILMs degenerate to the stable zone boundary mode. In other words, ILMs are generated through the bifurcation from the zone boundary mode under a critical total energy $H$. It is called as "the tangent bifurcation" [16].

On the other hand, "the saddle-node bifurcation", which is shown in this Letter, occurs even in the high energy limit. Then the harmonic potential ratio $\alpha$ is not significant parameter for the saddle-node bifurcation. Therefore the stability of ILM substantially depends on the nonlinear potential ratio $\beta$ in the cantilever array. 


\section{Conclusions}

We investigate the coexistence and stability of ILM in the micro-cantilever array, which is described by the coupled ordinary differential equations. As a result, it is shown that the quartic potentials ratio governs the stability of coexisting ILMs. The stability of ILM generally depends on the symmetry of amplitude distribution in space [3]. In the Fermi-Pasta-Ulam- $\beta$ (FPU- $\beta$ ) lattice, which has quadratic and quartic inter-site potentials, the P-mode is stable and the other is unstable [17]. However, the ST-mode is stable in the Klein-Gordon lattice which has quadratic and quartic on-site potentials and linear coupling. The P-mode is unstable in this lattice. The micro-cantilever array has nonlinearity in both on-site and inter-site potentials [12]. Then STmodes are unstable when the inter-site nonlinearity is sufficiently smaller than the on-site nonlinearity. If the inter-site nonlinearity becomes dominant, the ST-modes are stable. Thus the stability change of ILM is caused by the ratio in inter- and on-site nonlinearity. We identify that the stability changes occur through the saddle-node bifurcation. The bifurcation is not significantly depended on the harmonic terms. Because the bifurcation occurs even in the high energy limit.

Asymmetric ILMs are obtained in the region where all coexisting P- and STmodes are unstable. Their amplitude distribution has no symmetry. These ILMs are not classified into ST- or P-modes. In the region $\beta_{\mathrm{ST}} \leq \beta \leq \beta_{\mathrm{P}}$, twice number of ILMs coexist. If the coupled cantilever array is precisely fabricated, the asymmetric ILM should be excited experimentally. However the region is quite narrow. Analysis in the array with fixed boundaries suggests that the region is sensitive against impurities. Thus the excitation of asymmetric ILMs 
seems to be difficult.

The influence of impurities is enhanced near the critical value of the nonlinearity ratio. It is confirmed by comparing the ringed array to the edge-fixed array. In the ringed array, all coexisting ST-modes simultaneously lose their stability at $\beta=\beta_{\mathrm{ST}}$ because of the translational symmetry. The fixed boundaries, which are equivalent to quite heavy impurities, break the symmetry of the array. As a result, the stability change is not simultaneously caused. The parameter gaps between stable and unstable ILMs are emerged. Even if an ILM stands near the center of the array, the parameter gap exists. In other words, the influence of impurity against ILM is enhanced near the stability change of ILM.

The nonlinearity ratio is determined by the design of the array. Then the stability of ILM depends on the design of the cantilever array. It implies that the spatial symmetry of ILM in MEMS devices is selectable by the design of the devices. In addition, if the ratio is adjustable in time, the stability will be controllable. Applications using ILM would be realized by stability switching.

\section{Acknowledgements}

We would like to show our appreciation to Professor M. Sato, Kanazawa University, Japan, for the discussion about the model of cantilever array. This research was partially supported by the Ministry of Education, Culture, Sports, Science and Technology in Japan, The 21st Century COE Program No. 14213201 and the Global-COE program. 


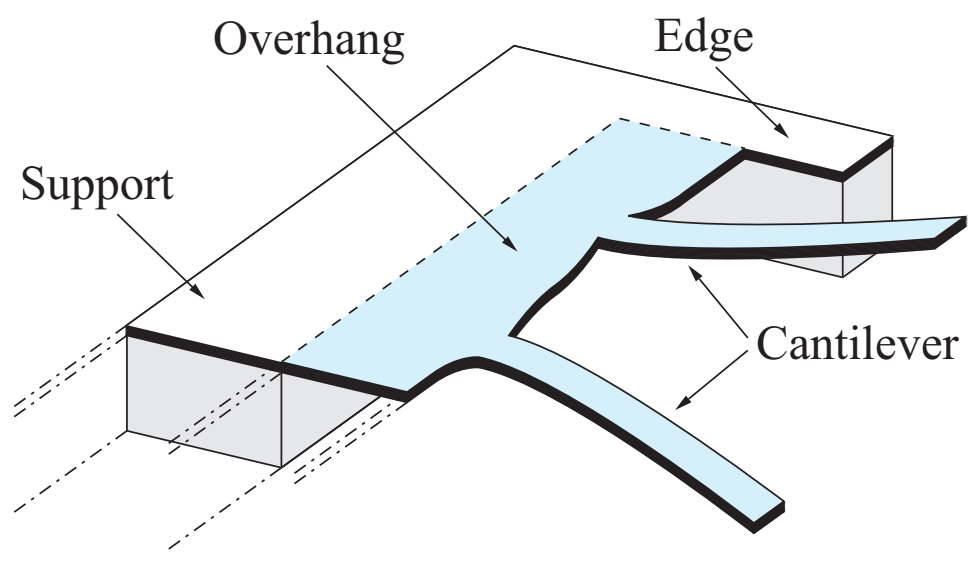

Fig. 1. Schematic configuration of cantilever array. The array consists of eight cantilevers, the overhang part, and the support. The interacting force between adjacent cantilevers is caused by bending of the overhang part.

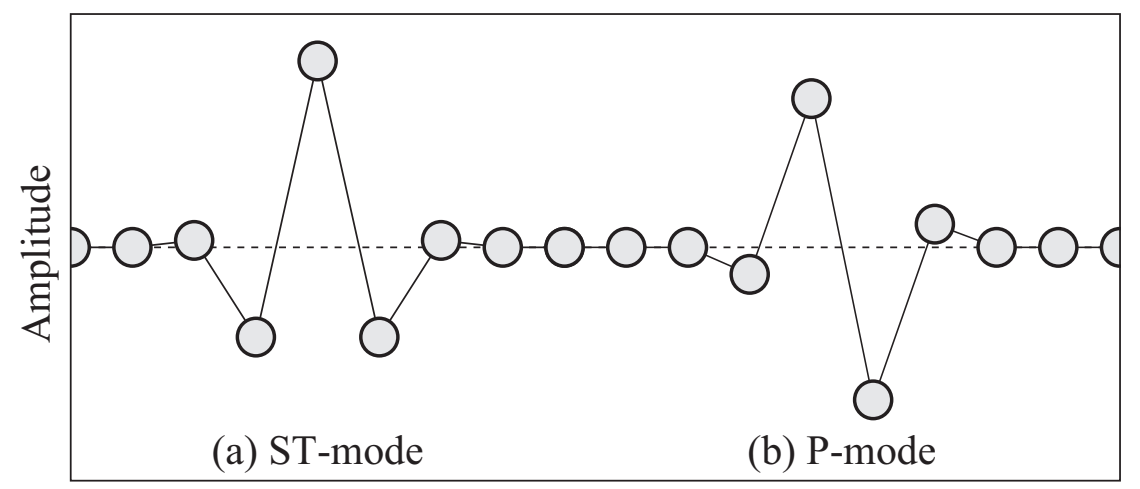

Fig. 2. Sievers-Takeno (ST) mode (a) and Page (P) mode (b). Each circle indicates the position of oscillator. Each line between circles corresponds the schematic coupling connection. Circles on the horizontal dashed line will not show the remarkable oscillation. The large displacement implies that the oscillator shows the periodic oscillation with large amplitude.

\section{References}

[1] A. J. Sievers, S. Takeno, Intrinsic localized modes in anharmonic crystals, Phys. Rev. Lett. 61 (1988) 970.

[2] S. Flach, A. Gorbach, Discrete breathers in fermi-pasta-ulam lattices, Chaos 15 

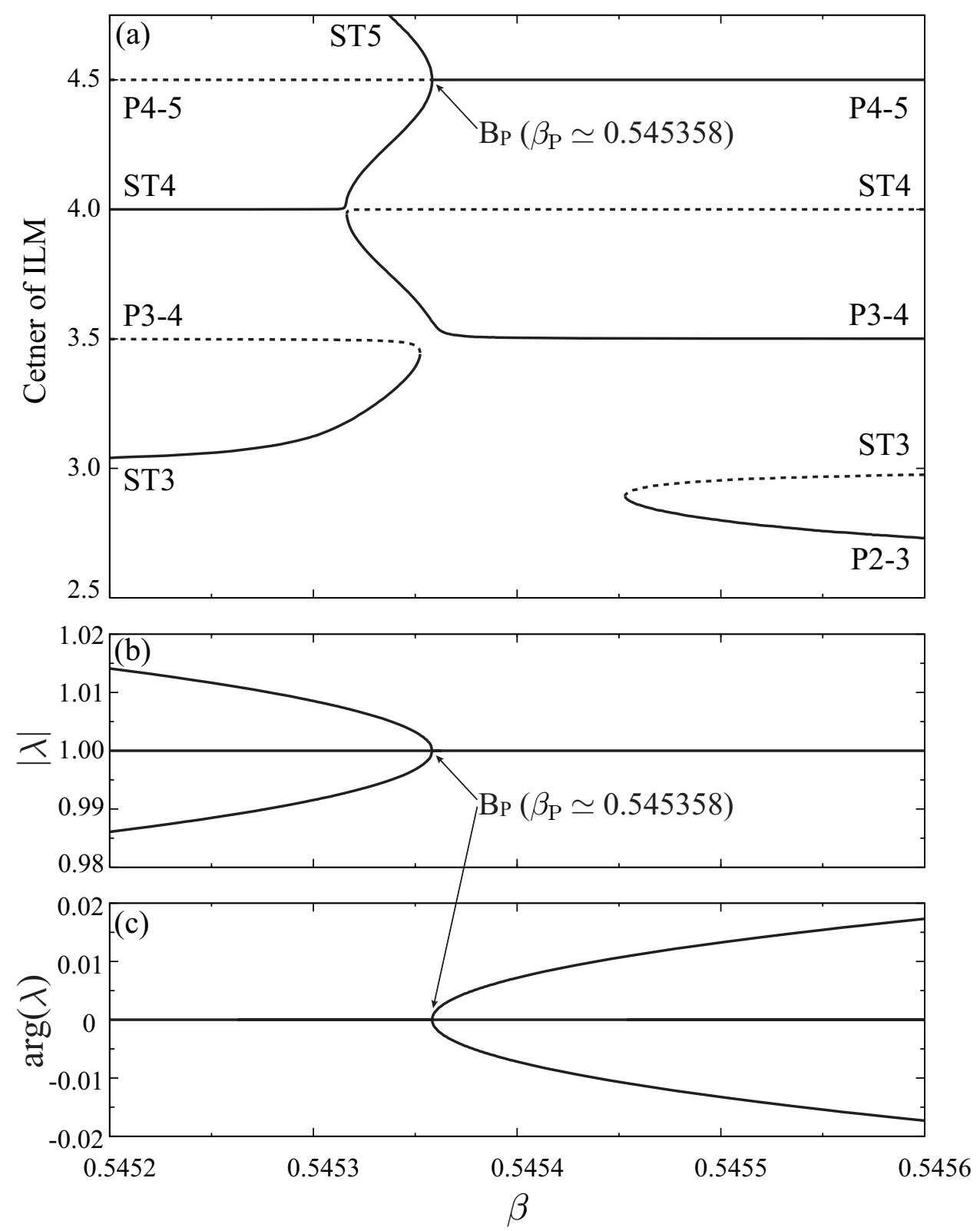

Fig. 3. Stability change of ILMs to the ratio $\beta$ between nonlinear on-site and inter-site potentials with the harmonic potential ratio $\alpha=0.1$. (a) Position and stability of ILM. Solid curve is a set of the marginally stable ILM. Namely, all Floquet multipliers are on unit circle in complex plane. Dashed curves correspond to the unstable ILMs. Absolute value (b) and argument (c) of Floquet multipliers of P4-5. The saddle-node bifurcation occurs at $\beta \simeq 0.545358$. 

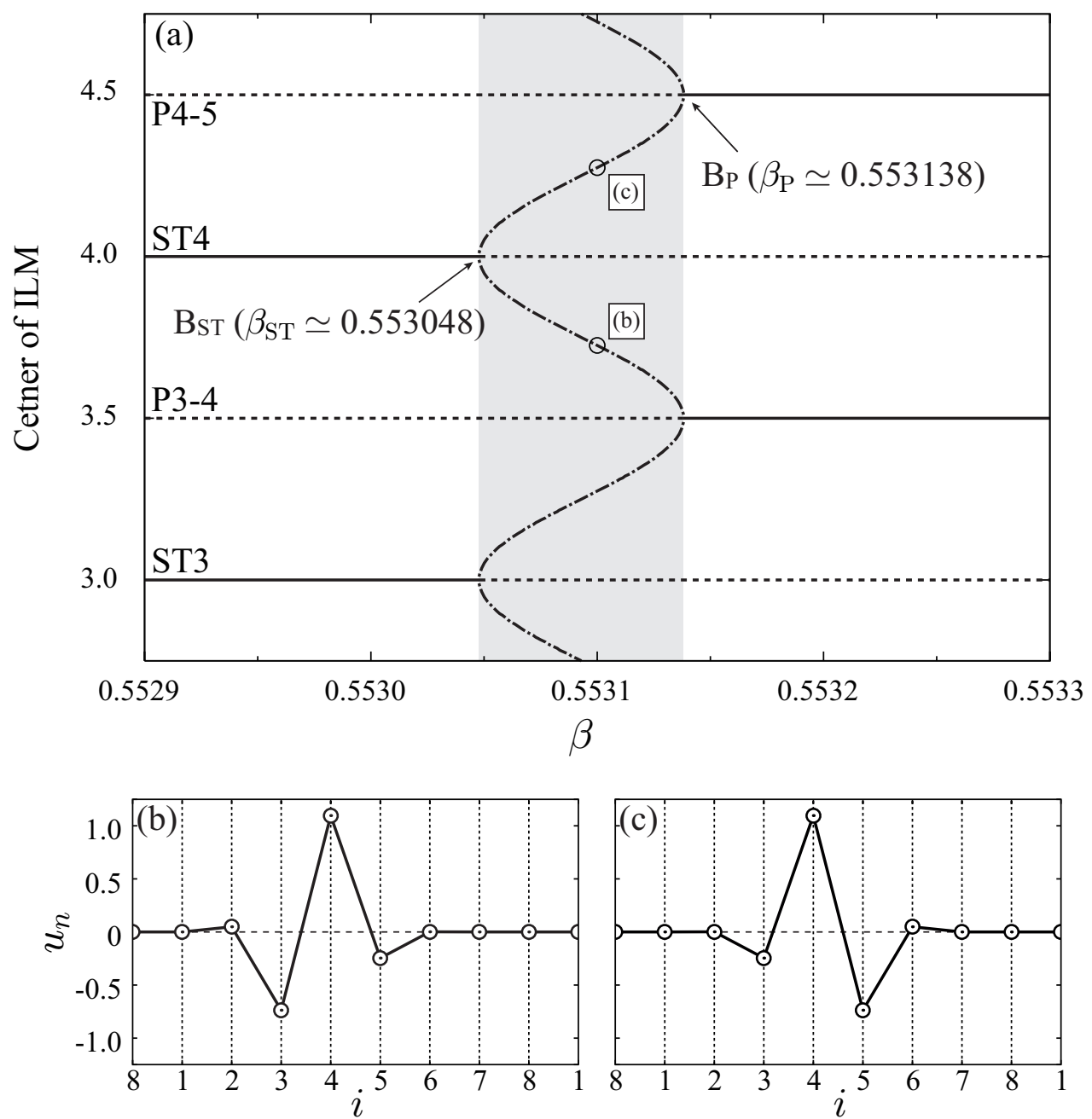

Fig. 4. (a) Stability change of ILMs with respect to $\beta$ in a ringed cantilever array without harmonic potentials. Solid line and dashed line represent marginally stable and unstable ILM, respectively. Dash-dotted curves correspond to marginally stable asymmetric ILMs. The asymmetric ILMs exist in the darkly hatched area, where ST-modes and P-modes are unstable. (b) and (c) Amplitude distribution of asymmetric ILMs.

(2005) 15112.

[3] S. Flach, C. R. Willis, Discrete breathers, Phys. Rep. 295 (1998) 181.

[4] E. Trías, J. J. Mazo, T. P. Orlando, Discrete breathers in nonlinear lattices: Experimental detection in a josephson array, Phys. Rev. Lett. 84 (2000) 741. 
[5] P. Binder, D. Abraimov, A. V. Ustinov, S. Flach, Y. Zolotaryuk, Observation of breathers in josephson ladders, Phys. Rev. Lett. 84 (2000) 745.

[6] A. V. Ustinov, Imaging of discrete breathers, Chaos 13 (2003) 716.

[7] H. S. Eisenberg, Y. Silberberg, R. Morandotti, A. R. Boyd, J. S. Aitchison, Discrete spatial optical solitons in waveguide arrays, Phys. Rev. Lett. 81 (1998) 3383.

[8] R. Morandotti, U. Peschel, J. S. Aitchison, H. S. Eisenberg, Y. Silberberg, Dynamics of discrete solitons in optical waveguide arrays, Phys. Rev. Lett. 83 (1999) 2726.

[9] M. Sato, B. E. Hubbard, A. J. Sievers, B. Ilic, D. A. Czaplewski, H. G. Craighead, Observation of locked intrinsic localized vibrational modes in a micromechanical oscillator array, Phys. Rev. Lett. 90 (2003) 044102.

[10] D. K. Campbell, S. Flach, Y. S. Kivshar, Localizing energy through nonlinearity and discreteness, Phys. Today 57 (2004) 43.

[11] M. Sato, B. E. Hubbard, L. Q. English, A.J.Sievers, B. Ilic, D. A. Czaplewski, H. G. Craighead, Study of intrinsic localized vibrational modes in micromechanical oscillator arrays, Chaos 13 (2003) 702.

[12] M. Sato, B. E. Hubbard, A. J. Sievers, Colloquium: nonlinear energy localization and its manipulation in micromechanical oscillator arrays, Rev. Mod. Phys. 78 (2006) 137.

[13] M. Sato, B. E. Hubbard, A. J. Sievers, B. Ilic, H. G. Craighead, Optical manipulation of intrinsic localized vibrational energy in cantilever arrays, Europhys. Lett. 66 (3) (2004) 318.

[14] J. B. Page, Asymptotic solutions for localized vibrational modes in strongly anharmonic periodic systems, Phys. Rev. B 41 (1990) 7835. 
[15] P. Bergé, Y. Pomeau, C. Vidal, Order within Chaos, Wiley, New York, 1984.

[16] S. Flach, Tangent bifurcation of band edge plane waves, dynamical symmetry breaking and vibrational localization, Physica D 91 (1996) 223.

[17] K. W. Sandusky, J. B. Page, K. E. Schmidt, Stability and motion of intrinsic localized modes in nonlinear periodic lattices, Phys. Rev. B 46 (10) (1992) 6161. 